\title{
Status of Cardiac Surgical Intensive Care Medicine in Germany in 2018: A Report on Behalf of the German Society for Thoracic and Cardiovascular Surgery
}

\author{
${ }^{1}$ Deutsche Gesellschaft für Thorax-, Herz- und Gefäßchirurgie \\ [DGTHG], Berlin, Germany \\ 2 Klinik für Herz- und Gefäßchirurgie, Universitäts-Herzzentrum \\ Freiburg Bad Krozingen Standort Freiburg, Freiburg, Baden- \\ Württemberg, Germany
}

Andreas Markewitz ${ }^{1}$ Jana Lewandowski ${ }^{1}$ Georg Trummer ${ }^{2}$ Andreas Beckmann ${ }^{1}$

Thorac Cardiovasc Surg 2020;68:377-383.

\begin{abstract}
Keywords

- cardiac surgery

- intensive care medicine

- survey

- data

- quality

Background This report summarizes the results of a voluntary survey designed to assess the current situation of cardiac surgical intensive care medicine in Germany in 2018.

Methods A standardized questionnaire concerning detailed information about structural characteristics of cardiac surgical intensive care units (ICUs) was sent to all German departments performing cardiac surgery.

Results Response rate was 93\% $(n=75 / 81)$. Compared with previous surveys since 1998 , the median number of intensive care beds for patients after cardiac surgery increased from 15 in 2013 to 16 in 2018. The proportion of cardiac surgical ICUs decreased to $51 \%$ with a simultaneous increase of interdisciplinary ICUs. The proportion of cardiac surgeons acting as director of an ICU increased to 43\%. The physicians' teams were mostly interdisciplinary (57\%). More than half of the directors were boardcertified intensivists (62\%) with a peak of $100 \%$ in ICUs run by cardiac surgeons. Human resources development in the ICU showed similar trends with an increase of physicians and nurses. More than half of all ICUs (61\%) and the vast majority of cardiac surgical ICUs (82\%) offer an accredited training program for intensive care medicine.

Conclusion The results of this survey corroborate once again that intensive care medicine represents a substantial and important part of cardiac surgery. However, efforts are necessary to keep this attitude alive for the future.
\end{abstract}

\author{
Address for correspondence Andreas Markewitz, MD, PhD Col (ret.), \\ Deutsche Gesellschaft für Thorax-, Herz- und Gefäßchirurgie \\ [DGTHG], Luisenstr. 58-59, D 10117 Berlin, Germany \\ (e-mail: AndreasMarkewitz@gmx.de).
}

\section{Introduction}

Intensive care medicine is an integral part of cardiac surgery. The availability of intensive care capacities determines operative volume, while the quality of intensive care medicine significantly contributes to the outcome of patients after cardiac operations. Thus, the proportion of intensive care resources related to the total bed capacity of the department is highest for cardiac surgery compared with other surgical specialties in Germany. ${ }^{1}$

received

May 27, 2019

accepted

May 28, 2019

published online

July 12, 2019 pitals led to the common belief of hospital business management that best practice for postoperative intensive care medicine should be realized on large inter- or multidisciplinary units, frequently under anesthesiological management. This attitude is somewhat inconsistent with the educational process of a cardiac surgical intensivist as well as with the empiric knowledge of experienced intensive care physicians that special challenges require specific skills and experience. In addition, the anticipated and in some

(c) 2020 Georg Thieme Verlag KG Stuttgart · New York
DOI https://doi.org/ 10.1055/s-0039-1693028. ISSN 0171-6425. 
departments, already existing reduction of residents and fellows in cardiac surgery results in a manpower shortage with concentration of resources for the operating room, while recruitment of qualified trainees for perioperative patient treatment, for example, for intensive care medicine, remains a relevant challenge.

Therefore, it was the objective of this assessment to gain actual information about the status of cardiac surgical intensive care medicine in Germany in 2018 and to compare the actual results with those of the preceding four surveys since 1998.

\section{Methods}

In summer 2018, a standardized questionnaire was sent by mail to all departments performing cardiac surgery in Germany (-Table 1). Initial nonresponders received two additional reminders. Answers were collected using a free online survey tool (SurveyMonkey; SurveyMonkey Europe UC, 2nd Floor, 2 Shelbourne Buildings, Shelbourne Road, Dublin, Ireland).

\section{Results}

Seventy-five departments with intensive care units (ICUs) for perioperative cardiac surgical care returned the completed questionnaire, resulting in a response rate of $93 \%$.

\section{Structure of ICU and Intermediate Care Units}

The total number of intensive care beds available for cardiac surgical patients in the responding institutions decreased from 1.404 in 2013 to 1.324 in 2018 . However, due to the lower response rate in 2018 , the number of intensive care beds in all cardiac surgical departments in Germany must be presumed to be actually higher. This corresponds to the slight increase in the mean and median number of beds (-Table 2). Looking at the ICU dimensions in more detail, a steady decrease of small units, that is, ICUs with less than 15 beds, can be observed. In contrast, the number of larger units increased from 1999 until 2008 with no change in 2013 but a slight increase in 2018 (-Fig. 1).

The number of isolated cardiac surgical ICUs decreased from $77 \%(n=51 / 66)$ in 1998 to $51 \%(n=38 / 75)$ in 2018 , whereas the number of interdisciplinary ICU increased (-Fig. 2).

Intermediate care (IMC) or step-down units were available in 51 hospitals (72\%). The number and rate of IMC units showed only small variations over the observed time period (1998: $n=49 / 66$ [74\%], 2003: $n=56 / 73$ [77\%], 2008: $n=51 / 73$ [70\%], and 2013: $n=58 / 81$ [71\%]). In contrast, the mean and median number of IMC beds constantly increased over time ( - Table 3 ).

\section{ICU Management}

In 2018, 32 ICUs (43\%) were managed by cardiac surgeons. This represents a higher rate than in 2013 when the nadir was observed. As in 2013, more ICUs $(n=34 / 75=45 \%)$ are managed by anesthesiologists ( - Fig. 3 ). The number and rate of ICUs managed by others, for example, by an interdisciplinary team, decreased to $12 \%$.
Table 1 Questionnaire

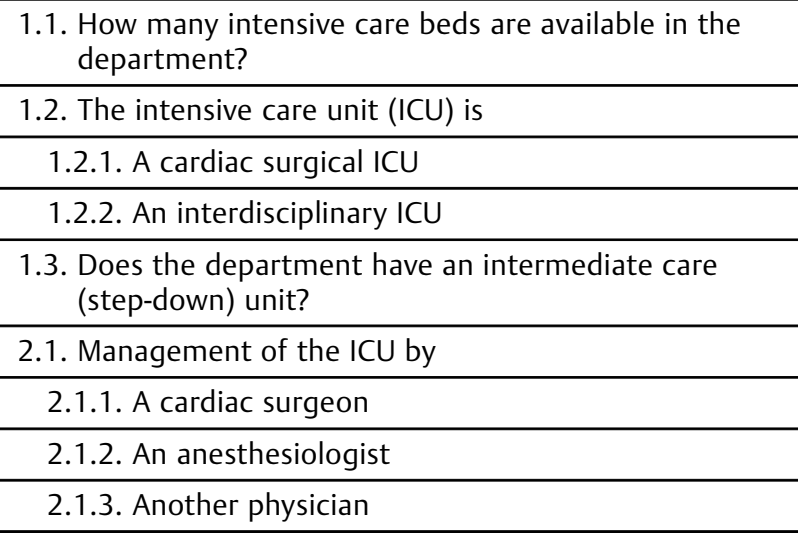

2.2. The head of the ICU is a board-certified cardiac surgical intensivist

2.3. The head of the ICU is exclusively responsible for the intensive care unit

2.4. The head of the ICU decides independently about the availability of intensive care capacities

2.5. Who takes care of the patients in the ICU?

2.5.1. Cardiac surgeons (residents and/or consultants)

2.5.2. Anesthesiologists (residents and/or consultants)

2.5.3. Both

2.5.4. Others

3.1. How many physicians (residents and/or consultants) are working on the ICU (full-time equivalent)?

3.1.1. How many staff members are board-certified cardiac surgical intensivists?

3.2. How many nurses are working on the ICU (full-time equivalent)?

3.2.1. How many of these staff members are board-certified ICU nurses?

3.3. How many additional staff are working in the ICU?

4. Does the department have the authorization for advanced training in cardiac surgical intensive care medicine?

4.1. If yes, for which advanced training period

5. Are there multidisciplinary meetings in the ICU and which specialty does participate?

5.1. Microbiology

5.2. Hygiene

5.3. Pharmacy

5.4. Physiotherapy

5.5. Dietetics

5.6. Others

6. Are standard operating procedures and/or clinical pathways introduced in the ICU?

The number of board-certified ICU directors as well as their responsibilities and privileges are given in - Table 4: the number of board-certified ICU directors increased, in particular, in cardiac surgical ICUs, reaching $100 \%$ in those 
Table 2 Number of intensive care beds in Germany at different points of time

\begin{tabular}{|l|l|l|l|l|l|}
\hline Number of beds & $\begin{array}{l}\mathbf{1 9 9 8} \\
(\boldsymbol{n}=\mathbf{6 6})\end{array}$ & $\begin{array}{l}\mathbf{2 0 0 3} \\
(\boldsymbol{n}=\mathbf{7 3})\end{array}$ & $\begin{array}{l}\mathbf{2 0 0 8} \\
(\boldsymbol{n}=\mathbf{6 5 )}\end{array}$ & $\begin{array}{l}\mathbf{2 0 1 3} \\
(\boldsymbol{n}=\mathbf{8 1})\end{array}$ & $\begin{array}{l}\mathbf{2 0 1 8} \\
(\boldsymbol{n}=\mathbf{7 5})\end{array}$ \\
\hline Total & 817 & 1,020 & 1,177 & 1,404 & 1,324 \\
\hline Mean & 12.38 & 13.97 & 18.11 & 17.33 & 18.14 \\
\hline SD & 7.40 & 7.34 & 11.08 & 9.26 & 9.16 \\
\hline Median & 11 & 12 & 15 & 15 & 16 \\
\hline Minimum & 5 & 5 & 6 & 5 & 6 \\
\hline Maximum & 50 & 46 & 70 & 51 & 60 \\
\hline
\end{tabular}

Abbreviation: SD, standard deviation.

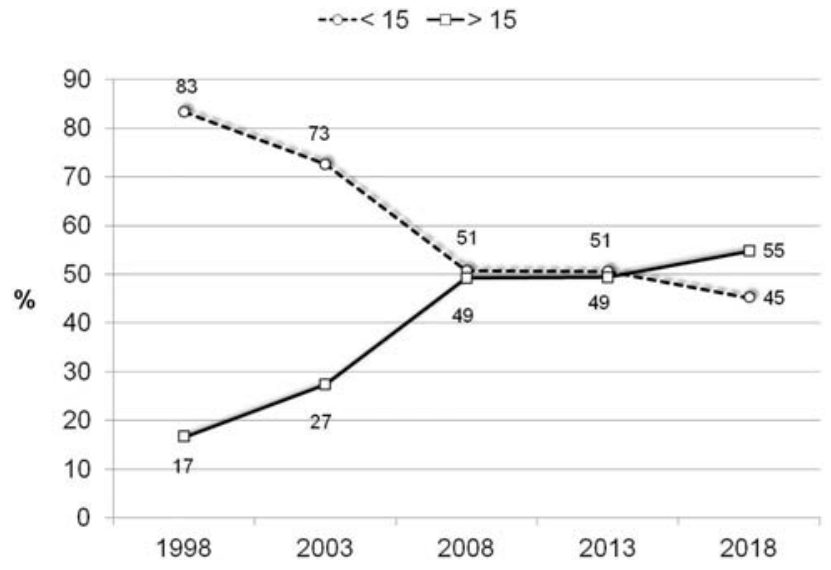

Fig. 1 Rate of cardiac surgical ICU with 15 or less beds compared with ICU with more than 15 beds over time. ICU, intensive care unit.

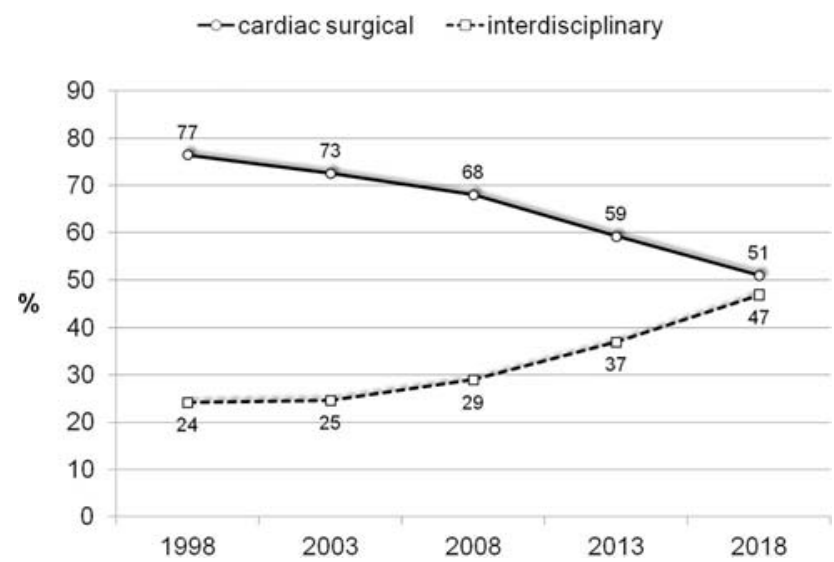

Fig. 2 Type of intensive care unit for cardiac surgical patients.

Table 3 Intermediate care beds in Germany 1998 to 2018

\begin{tabular}{|l|l|l|l|l|l|}
\hline IMC beds & $\mathbf{1 9 9 8}$ & $\mathbf{2 0 0 3}$ & $\mathbf{2 0 0 8}$ & $\mathbf{2 0 1 3}$ & $\mathbf{2 0 1 8}$ \\
\hline Total & 458 & 558 & 552 & 729 & 687 \\
\hline Mean & 9.35 & 9.96 & 10.62 & 12.57 & 12.72 \\
\hline SD & 6.94 & 7.42 & 8.60 & 8.80 & 7.30 \\
\hline Median & 7 & 8 & 8 & 10 & 12 \\
\hline Minimum & 3 & 1 & 1 & 2 & 4 \\
\hline Maximum & 32 & 32 & 44 & 56 & 40 \\
\hline
\end{tabular}

Abbreviations: IMC, intermediate care; SD, standard deviation.

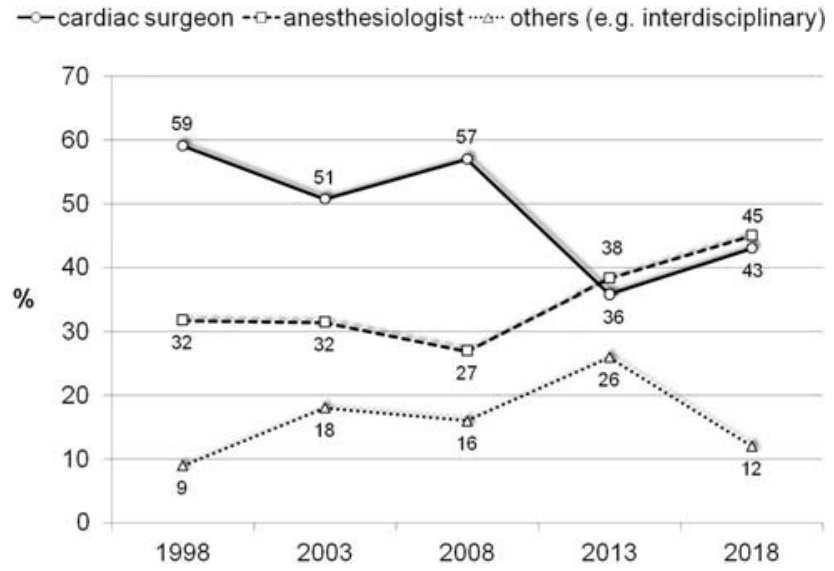

Fig. 3 Specialty of the director of intensive care unit.

directed by a cardiac surgeon. This applies as well to the number of ICU directors with no other responsibilities than managing the ICU. Finally, more than two-thirds of the ICU directors manage ICU capacities independently.

The specialty of the physicians working at the bedside of ICU patients is shown in - Fig. 4. As compared with 2013, the multi- or interdisciplinary model has decreased, and monodisciplinary solutions have gained importance.

\section{Medical Staff and Qualification}

The results for the medical staff on cardiac surgical ICUs show that there is an increase both for physicians and for nurses (-Table 5). For the physician-to-bed ratio (-Fig. 5) and the nurse-to-bed ratio ( - Fig. $\mathbf{6}$ ), the same trend was observed.

- Table 6 shows the number of cardiac surgical centers in which board-certified physicians as well as board-certified nurses are working. In addition, the total number of boardcertified ICU physicians and nurses are given. The total number of departments with board-certified ICU physicians has increased over time, while the rate of board-certified ICU nurses remained constant on a high and even rising level over the observed period, reaching almost 50\% in 2018 (-Fig. 7).

Additional staff, such as study nurses or coding specialists, is available in 63 of $75 \mathrm{ICU}$ (84\%). The total number of this personnel increased over time from $2.0 \pm 1.3$ in 2003 to $2.3 \pm 2.6$ in 2008 and $2.8 \pm 2.3$ in 2013 to $3.7 \pm 3.4$ in 2018 . 
Table 4 Board-certified ICU directors, responsibilities and management

\begin{tabular}{|c|c|c|c|c|c|c|c|c|}
\hline & \multicolumn{2}{|c|}{2003} & \multicolumn{2}{|c|}{2008} & \multicolumn{2}{|c|}{2013} & \multicolumn{2}{|c|}{2018} \\
\hline & $n$ & $\%$ & $n$ & $\%$ & $n$ & $\%$ & $n$ & $\%$ \\
\hline Board-certified intensivist & 35 & 48 & 33 & 51 & 44 & 54 & 45 & 62 \\
\hline In cardiac surgical ICUs & 29 & 55 & 30 & 68 & 32 & 67 & 32 & 82 \\
\hline In cardiac surgical ICUs directed by a cardiac surgeon & 24 & 69 & 28 & 78 & 22 & 81 & 32 & 100 \\
\hline Responsibility for ICU management only & 38 & 52 & 31 & 48 & 51 & 63 & 48 & 65 \\
\hline In cardiac surgical ICUs & 21 & 57 & 18 & 50 & 31 & 65 & 28 & 72 \\
\hline Autonomous decision making & 51 & 70 & 46 & 71 & 54 & 68 & 50 & 68 \\
\hline In cardiac surgical ICUs & 34 & 92 & 33 & 92 & 34 & 71 & 27 & 69 \\
\hline
\end{tabular}

Abbreviation: ICU, intensive care unit.

$-\infty$ cardiac surgeon $\quad-\infty \cdot$-anesthesiologist $\quad \cdots \cdot-\cdot$ interdisciplinary

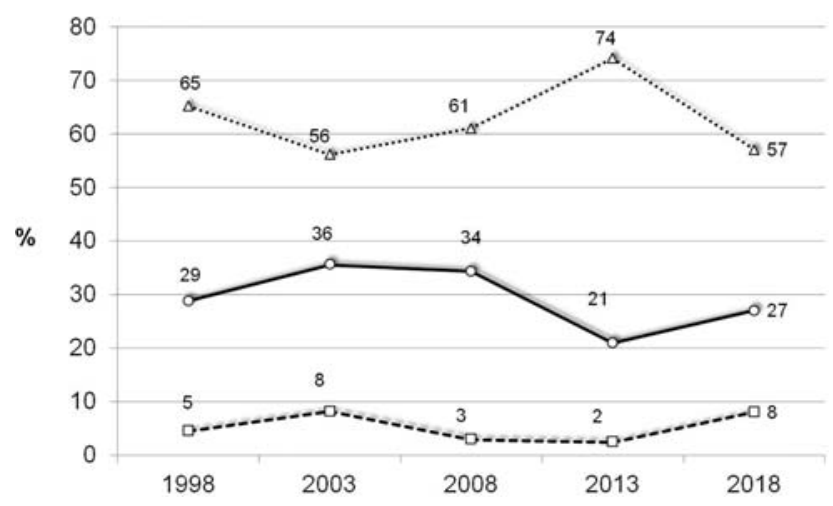

Fig. 4 Specialty of intensive care unit physicians.

\section{Advanced Training Authorization, Multidisciplinary Rounds, and Standard Operating Procedures}

An authorized intensivist with a license to train physicians in surgical intensive care medicine was available on 45 ICUs. This number is higher than in previous years (1998: $n=29$, 2003: $n=39,2008: n=39$, and 2013: $n=40$ ). In addition, the total rate of ICUs, on which an authorized physician for training of intensivists is available, increased in general, and was highest on cardiac surgical ICUs, in particular, if they were directed by a cardiac surgeon ( - Table 7 ).

In 72 ICUs, multidisciplinary rounds were established. This is a higher absolute number and rate as compared with previous years (-Table 8). Microbiologists were the most common specialty involved, followed by physiotherapists, hygiene specialists, pharmacists, and others, for example, radiologists.

The absolute number of ICUs using standard operating procedures (SOP) initially decreased from 55 in 2003 to 53 in 2008 , then increased to 71 in 2013 , and is actually 71 . The corresponding rates are demonstrated in - Fig. 8 and show a steady increase of SOPs.

\section{Discussion}

The results of this survey underline the daily clinical experience that intensive care medicine is an important and integral part of the clinical pathway for patients undergoing cardiac surgery.

According to the most recent publication of the German Federal Office of Statistics, ${ }^{1}$ intensive care capacities with a total amount of 1,472 beds represented $28.5 \%$ of all beds available in cardiac surgical departments in Germany in 2017. Corresponding rates for other surgical specialties such as orthopaedic, trauma, vascular, abdominal, thoracic, and neurosurgery are $2.7,4.5,6.2,10.2,12.4$, and $14.7 \%$, respectively. $^{1}$ Therefore, cardiac surgical departments have 2 to 10 times higher proportion of intensive care beds at their disposal than the aforementioned surgical specialties, highlighting the paramount importance of intensive care medicine in cardiac surgery.

The continuous increase of cardiac surgical intensive care capacities in the context of the slowly decreasing number of cardiac operations in Germany over more than a decade ${ }^{2}$ can be explained by various factors such as an increase of patients' age leading to a growing number of comorbidities

Table 5 Number of physicians and nurses working in the ICU as mean \pm SD and median

\begin{tabular}{|c|c|c|c|c|c|c|c|c|}
\hline & \multicolumn{4}{|c|}{ Physicians } & \multicolumn{4}{|l|}{ Nurses } \\
\hline & $\begin{array}{l}2003 \\
(n=73)\end{array}$ & $\begin{array}{l}2008 \\
(n=65)\end{array}$ & $\begin{array}{l}2013 \\
(n=81)\end{array}$ & $\begin{array}{l}2018 \\
(n=75)\end{array}$ & $\begin{array}{l}2003 \\
(n=73)\end{array}$ & $\begin{array}{l}2008 \\
(n=65)\end{array}$ & $\begin{array}{l}2013 \\
(n=81)\end{array}$ & $\begin{array}{l}2018 \\
(n=75)\end{array}$ \\
\hline Mean & 6.0 & 7.4 & 9.2 & 9.9 & 40.6 & 55.3 & 51.6 & 56.8 \\
\hline SD & 4.4 & 6.2 & 8.0 & 8.1 & 18.4 & 30.2 & 33.4 & 32.6 \\
\hline Median & 5 & 6 & 7 & 8 & 37 & 46 & 40 & 44 \\
\hline
\end{tabular}

Abbreviations: ICU, intensive care unit; SD, standard deviation. 


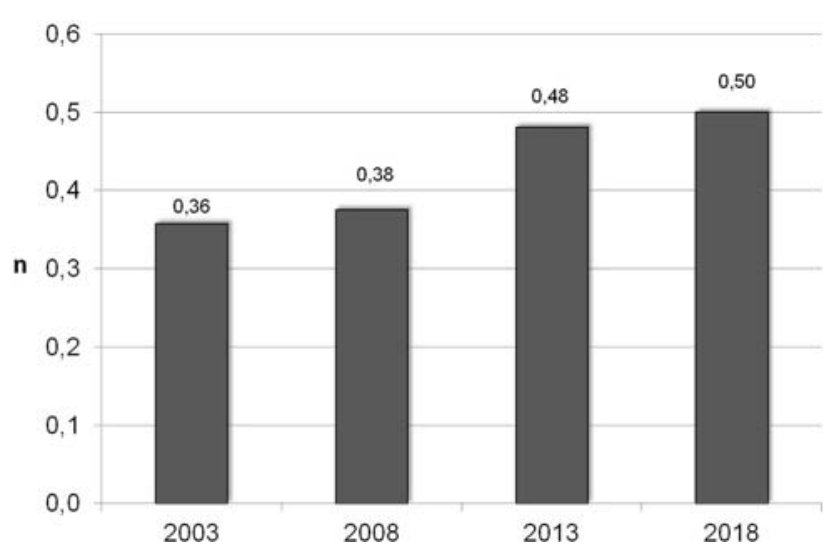

Fig. 5 Physician-to-bed ratio (median).

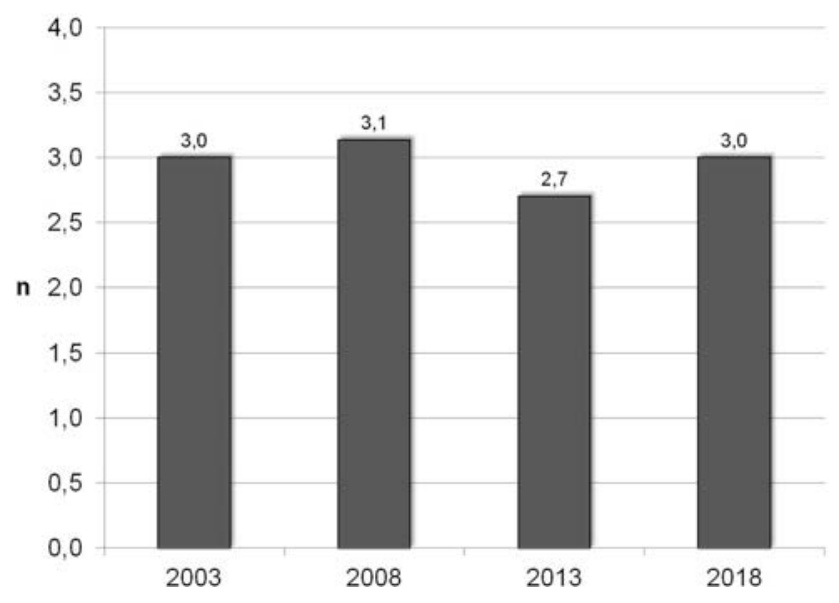

Fig. 6 Nurse-to-bed ratio (median).

and resulting in higher risk profiles. In addition, the increase of patients suffering from acute or chronic heart failure, requiring for example, operations for temporary or longterm mechanical circulatory support, implies an additional demand for intensive care resources.

Several economic factors, for example, cost pressure and improvements in efficient resource management, have induced the development of organizational concepts with a concentration of ICU capacities in large multidisciplinary units since the early 2000s. Usually, these interdisciplinary ICUs are more flexible in allocation of available capacities and staff assignment, thus optimizing critical care resource utilization. As a result, the number and rate of ICUs with more than 15 beds increased substantially until 2008 remained constant in 2013 and showed an increase in 2018 again.

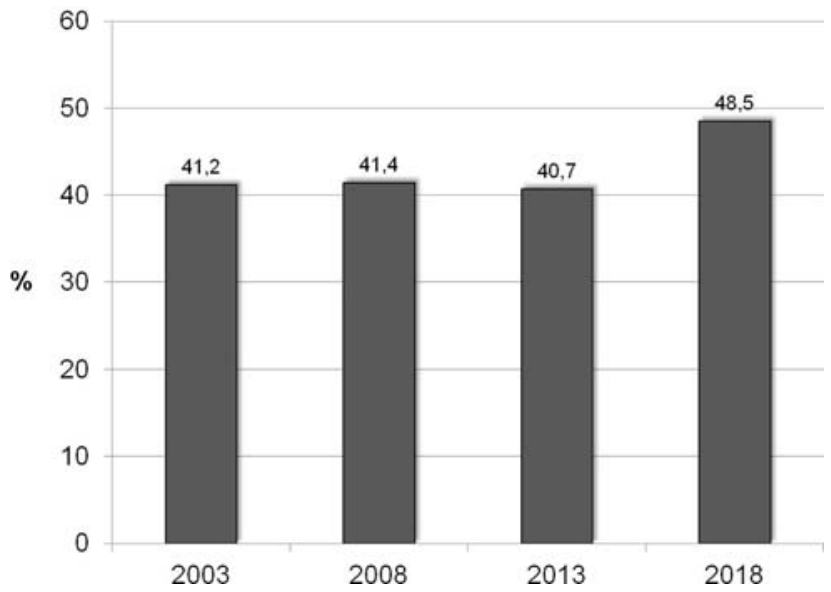

Fig. 7 Rate of board-certified nurses.

The number and rate of interdisciplinary ICUs increased further in 2018 as compared with previous surveys. Since the decision processes include more than one responsible person or a single medical specialty, diverging goals in the management of interdisciplinary units may sometimes compromise the availability of ICU capacities for cardiac surgical patients. However, for a given multimorbid patient, the multidisciplinarity may result in a benefit, when the experience of several intensivists from different specialties can be brought together.

In many hospitals, IMC units have been established to increase flexibility in allocation of critical care resources. The growing demand for these resources is reproducible by the continuously increasing number of IMC beds in cardiac surgery.

However, it is somewhat surprising that, in contrast to the total number of beds, the number of cardiac surgical departments with IMC units did not really increase since 1998, indicating that hospital managers follow different strategies to handle their intensive care resources.

Comprehensive statistics about cardiac surgical ICUs are not available in the literature; therefore, a comparison of our data to other national or international surveys is impossible. However, it is reported that patients having undergone coronary artery bypass grafting or heart valve operations represent the biggest group of adult patients treated on ICUs in Canada in 2013/2014, ${ }^{3}$ confirming the outstanding importance of available ICU capacities for departments performing cardiac surgery.

Another consequence of merging ICUs until 2013 was the decreasing number of cardiac surgeons holding the position of the ICU director, since interdisciplinary ICUs are usually managed by anesthesiologists. By 2018, the rate of cardiac surgical directors increased again as did the rate of

Table 6 Board-certified ICU physicians and/or ICU nurses

\begin{tabular}{|l|l|l|l|l|l|l|l|l|}
\hline & \multicolumn{3}{|l|}{ Board-certified physicians } & \multicolumn{3}{l|}{ Board-certified nurses } \\
\cline { 2 - 9 } & 2003 & 2008 & 2013 & 2018 & 2003 & 2008 & 2013 & 2018 \\
\hline Institutions & $44 / 73$ & $48 / 65$ & $53 / 81$ & $63 / 75$ & $65 / 73$ & $57 / 65$ & $71 / 81$ & $65 / 75$ \\
\hline Number & 85 & 134 & 156 & 260 & 1,079 & 1,209 & 1,571 & 1,411 \\
\hline
\end{tabular}

Abbreviation: ICU, intensive care unit. 
382 Status of Cardiac Surgical Intensive Care Medicine in Germany in 2018 Markewitz et al.

Table 7 Percentage of ICUs with licensed authorization to train physicians in surgical intensive care medicine

\begin{tabular}{|c|c|c|c|c|c|}
\hline ICUs & $\begin{array}{l}1999 \\
(n=66)\end{array}$ & $\begin{array}{l}2003 \\
(n=73)\end{array}$ & $\begin{array}{l}2008 \\
(n=65)\end{array}$ & $\begin{array}{l}2013 \\
(n=81)\end{array}$ & $\begin{array}{l}2018 \\
(n=75)\end{array}$ \\
\hline All & 43.9 & 53.4 & 60.0 & 49.4 & 60.0 \\
\hline Cardiac surgical ICU & \multirow[t]{2}{*}{ N/A } & 58.2 & 58.7 & 64.6 & 81.6 \\
\hline Directed by cardiac surgeon & & 57.4 & 55.8 & 52.5 & 93.5 \\
\hline
\end{tabular}

Abbreviations: ICU, intensive care unit; N/A, not available.

Table 8 ICUs with multidisciplinary rounds

\begin{tabular}{|l|l|l|l|l|l|l|l|}
\hline 2003 & \multicolumn{2}{l|}{2008} & \multicolumn{2}{l|}{2013} & \multicolumn{2}{l|}{2018} \\
\hline$n$ & $\%$ & $n$ & $\%$ & $n$ & $\%$ & $n$ & $\%$ \\
\hline $58 / 73$ & 80 & $50 / 65$ & 77 & $71 / 81$ & 88 & $72 / 75$ & 96 \\
\hline
\end{tabular}

Abbreviation: ICUs, intensive care units.

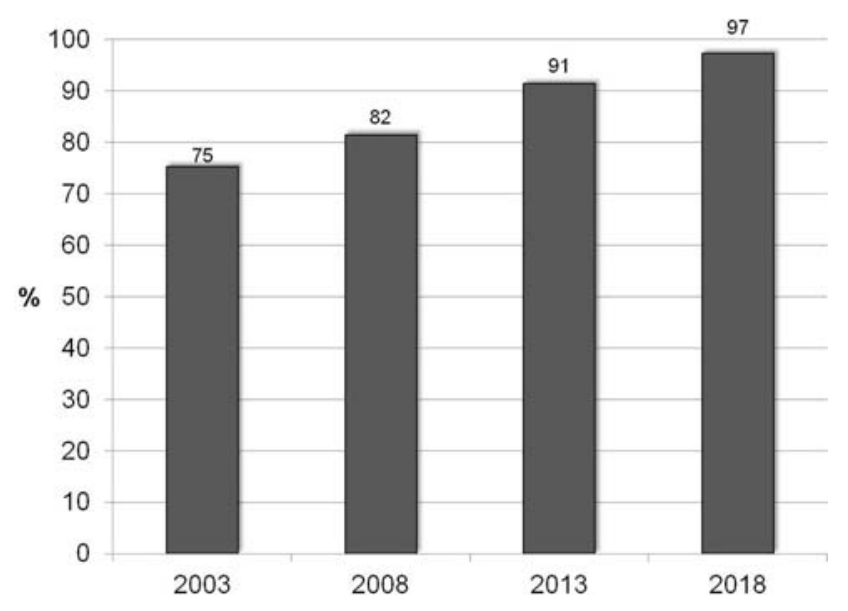

Fig. 8 Rate of intensive care units working according to standard operating procedures.

anesthesiologists directing the ICU for patients after cardiac operations. In contrast, the interdisciplinary leadership model has lost attraction compared with 2013. This shows that cardiac surgeons are willing and able to take responsibility for the complete perioperative treatment of their patients. The fact that the number and rate of institutions with board-certified ICU physicians ( $n=63 / 75=84 \%$ ) have substantially increased in 2018, as did the number of boardcertified ICU physicians $(n=260)$ supports this hypothesis.

It is somewhat surprising that not all ICU directors are board-certified intensivists, although the German reimbursement system for hospital treatment requires a board-certified ICU physician continuously directing and managing complex ICU therapies without any exception. We have no explanation for this observation. However, at least, all cardiac surgeons directing an ICU are board-certified intensivists.

As published by the German Interdisciplinary Association of Critical Care and Emergency Medicine (DIVI), an ICU director shall not have any other responsibility beside the management of the ICU. ${ }^{4}$ This requirement is fulfilled in only $65 \%$ of all ICUs and in only $72 \%$ of cardiac surgical ICUs. In addition, more than $30 \%$ of all ICU directors have to agree to compromises in some of their decisions about the availability or nonavailability of ICU capacities. Both results show no change but even a worsening as compared with previous surveys, and thus definitely require further improvements.

In contrast, the results for the staff on cardiac surgical ICUs show an improved trend compared with 2013, since the number of nurses as well as the nurse-to-bed ratio has increased again. The number of physicians as well as the physician-to-bed ratio shows a constant increase over time.

However, as in previous years, the requirements of the DIVI for an adequate physician-to-bed ratio are still not fulfilled despite the increasing number of ICU physicians, while the nurse-to-bed ratio fulfills the requirements compared with $2013 .{ }^{4}$ Additionally, the rate of board-certified ICU nurses is well above the requirements of the DIVI with a minimum level of $30 \%$. The increasing number of additional staff members seems to indicate the reaction of hospital executives for the growing administrative work to limit the time of ICU physicians and nurses for nonmedical issues. Taken together, these results still show some improvements of deficits in ICUs observed in previous years, leaving room for further improvements in the future, since there is good evidence for a substantial impact of staffing on the outcome of patients. ${ }^{5-7}$

A licensed training for the subspecialty surgical intensive care medicine can be completed in 45 of all ICUs (60\%) and in 31 of all specific cardiac surgical ICUs (78\%). Both results show an increase compared with previous surveys and, in addition to other results, underline the commitment and ability of cardiac surgeons to keep the skills to practice intensive care medicine.

Hopefully, this number and rate will remain high enough to fulfill the requirements for physician staffing on cardiac surgical ICUs some day.

Multidisciplinary rounds are known to improve the performance of an ICU and to improve the outcome of patients. ${ }^{8}$ Therefore, many institutions recommend multidisciplinary rounds, for example, the Institute for Healthcare Improvement ${ }^{9}$ or the American College of Critical Care Medicine. ${ }^{10}$ In addition, daily multiprofessional ward rounds with the documentation of therapy goals are 1 of 10 quality indicators of ICUs in Germany. ${ }^{11}$ The results of this survey clearly show that multidisciplinary rounds with physiotherapists, microbiologists, and hygiene nurses are common in German cardiac surgical ICUs, underlining the high quality of intensive care medicine for cardiac surgical patients.

SOPs were initially considered to exert beneficial outcome effects. However, in more recent publications, they have 
demonstrated conflicting results in critical care medicine: a recent Cochrane review showed a reduction of complications but no effect on readmission or in-hospital mortality. ${ }^{12}$ Another study even showed no impact of SOPs on outcome. ${ }^{13}$ Nevertheless, nearly all cardiac surgical ICUs had one or more SOPs to guide and standardize the therapy of their patients in 2018.

\section{Limitations}

This survey is subject to all limitations applying to results obtained by voluntary data collection and descriptive analyses from a standardized questionnaire. The validity of the data is monitored by rules for plausibility but not by an external audit. In addition, this and the preceding surveys had different response rates ranging from 81 to $100 \%$. All comparisons of the results of the current survey with preceding surveys as well as the interpretations based on these comparisons are therefore subject to a certain degree of uncertainty.

\section{Conclusion}

The results of this survey demonstrate once more ${ }^{14}$ that cardiac surgeons in Germany recognize the outstanding importance of intensive care medicine: First, a response rate of $93 \%$ for a voluntary survey can be considered as an indicator of the high priority of intensive care in cardiac surgery. Second, ICU capacities available for patients after cardiac operations constantly increase, and third, the majority of ICUs are specialized cardiac surgical ICUs. Finally, cardiac surgeons take care of their patients in nearly $100 \%$, either alone or as members of an interdisciplinary team.

The partial noncompliance with the recommendations of scientific medical societies regarding ICU staffing appears to be primarily the result of hospital managements' reaction to the economic pressure in health care systems, and it is certainly not based on limited interest of cardiac surgeons in intensive care medicine. Cardiac surgical departments in Germany prove daily that they have the competence to perform and educate intensive care medicine on a high-quality level. Therefore, the shortcomings outlined in this article have to be eliminated, and the attitude to understand that intensive care medicine is one of the key processes for patients in cardiac medicine has to be kept alive for the future.

Conflict of Interest

None.

\section{References}

1 Statistisches Bundesamt. Grunddaten der Krankenhäuser 2017 (September 2018). Available at: https://www.destatis.de/DE/Themen/Gesellschaft-Umwelt/Gesundheit/Krankenhaeuser/Publikatio nen/Downloads-Krankenhaeuser/grunddaten-krankenhaeuser-21 20611177004.pdf;jsessionid=3601B5606BAEA76414C707C9AA8 89A6B.internet731?_blob $=$ publicationFile $\& \mathrm{~V}=4$. Accessed April 16,2019

2 Beckmann A, Meyer R, Lewandowski J, Frie M, Markewitz A, Harringer W. German Heart Surgery Report 2017: The Annual Updated Registry of the German Society for Thoracic and Cardiovascular Surgery. Thorac Cardiovasc Surg 2018;66(08):608-621

3 Canadian Institute for Health Information. Care in Canadian ICUs, Available at: https://secure.cihi.ca/free_products/ICU_Report_ EN.pdf. Accessed April 24, 2019

4 Jorch G, Kluge S, König F, et al. Empfehlungen zur Struktur und Ausstattung von Intensivtherapiestationen - Kurzversion -. DIVI 2011;2:78-86

5 Neuraz A, Guérin C, Payet C, et al. Patient mortality is associated with staff resources and workload in the ICU: a multicenter observational study. Crit Care Med 2015;43(08):1587-1594

6 Driscoll A, Grant MJ, Carroll D, et al. The effect of nurse-to-patient ratios on nurse-sensitive patient outcomes in acute specialist units: a systematic review and meta-analysis. Eur J Cardiovasc Nurs 2018;17(01):6-22

7 Frost SA, Alexandrou E. Higher nurse staffing levels associated with reductions in unplanned readmissions to intensive care or operating theatre, and in postoperative in-hospital mortality in heart surgery patients. Evid Based Nurs 2013;16(02):62-63

8 Kim MM, Barnato AE, Angus DC, Fleisher LA, Kahn JM. The effect of multidisciplinary care teams on intensive care unit mortality. Arch Intern Med 2010;170(04):369-376

9 Improvement Map. Getting Started Kit: Multidisciplinary Rounds How-to Guide. Cambridge, MA: Institute for Healthcare Improvement; 2010. Available at: http://www.ihi.org/knowledge/Pages/Tools/HowtoGuideMultidisciplinaryRounds.aspx. Accessed October 20, 2013

10 Brilli RJ, Spevetz A, Branson RD, et al; American College of Critical Care Medicine Task Force on Models of Critical Care Delivery. The American College of Critical Care Medicine Guidelines for the Defintion of an Intensivist and the Practice of Critical Care Medicine. Critical care delivery in the intensive care unit: defining clinical roles and the best practice model. Crit Care Med 2001;29 (10):2007-2019

11 Kumpf O, Braun JP, Brinkmann A, et al. Quality indicators in intensive care medicine for Germany - third edition 2017. Ger Med Sci 2017; 15:Doc10

12 Rotter T, Kinsman L, James E, et al. Clinical pathways: effects on professional practice, patient outcomes, length of stay and hospital costs. Cochrane Database Syst Rev 2010;(03):CD006632

13 Sevransky JE, Checkley W, Herrera P, et al; United States Critical Illness and Injury Trials Group-Critical Illness Outcomes Study Investigators. Protocols and hospital mortality in critically ill patients: the United States Critical Illness and Injury Trials Group-Critical Illness Outcomes Study. Crit Care Med 2015;43(10):2076-2084

14 Markewitz A, Trummer G, Pilarczyk K, Beckmann A. Status of cardiac surgical intensive care medicine in Germany during 2013: a report on behalf of the German Society for Thoracic and Cardiovascular Surgery. Thorac Cardiovasc Surg 2014;62(07):536-542 